\title{
Prevalence of Exclusively Right-Sided Endomyocardial Fibrosis among Patients with Heart Failure in Equatorial Guinea
}

\author{
Marincheva-Savcheva Gergana ${ }^{1}$, Hazanov Eivgeny ${ }^{2}$, Neuberger Ami ${ }^{3, *}$, Garcia Maria Cecilia ${ }^{4}$, \\ Cesar Guarda Dario ${ }^{5}$ and Stamler Alon 6 \\ ${ }^{1,2}$ Department of Cardiology, ${ }^{3}$ Department of Internal Medicine, ${ }^{4,5}$ Department of Pediatrics ${ }^{6}$ Unit of Cardiothoracic \\ Surgery, Hospital La-Paz, Bata, Equatorial Guinea
}

\begin{abstract}
Endomyocardial fibrosis (EMF) is a common restrictive cardiomyopathy of unknown etiology, endemic to regions close to the equator. According to previously published reports, the disease affects only the right ventricle (RV) $10-28 \%$ of patients with endomyocardial fibrosis. The aims of this study were to determine the prevalence of EMF among patients with heart failure in Equatorial Guinea, and to describe its clinical manifestations. We used transthoracic echocardiography to determine the prevalence of EMF among 50 patients with newly diagnosed heart failure. We compared the demographic data, clinical features and eosinophilic counts in patients with EMF and patients with other forms of heart failure. During a study period of nine months ten patients were diagnosed with EMF, accounting for $20 \%$ of the patients diagnosed with heart failure. Curiously, all ten patients had exclusive right-sided disease. The average time from the onset of symptoms to diagnosis was 6.1 \pm 2.0 years (range 3-9 years), implicating a much better prognosis than previously reported. Eosinophil count was not higher in patients with EMF when compared to other patients with heart failure. The exclusive involvement of the right ventricle in ten consecutive patients, and the relatively long survival after the onset of symptoms, hint towards a specific variant of the disease in Equatorial Guinea.
\end{abstract}

Keywords: Endomyocardial fibrosis, heart failure, Africa

\section{INTRODUCTION}

Endomyocardial fibrosis (EMF) is a common, restrictive cardiomyopathy, endemic to regions close to the equator. It has no specific treatment and carries a poor prognosis, since most patients present with advanced heart failure [1-3]. The cause of EMF is still unknown. Several hypotheses linking infectious agents, toxins, or environmental factors to EMF's unique geographical distribution are yet to be corroborated [1,4]. According to previously published reports, the disease affects both ventricles in $50-55 \%$ of the patients, the left ventricle (LV) only in $17-40 \%$ of the patients, and exclusively the right ventricle $(R V)$ in the remaining $10-28 \%(2,5)$.

Since echocardiography was not available in Equatorial Guinea prior to 2007, the diagnosis of EMF was practically impossible. The incidence of EMF among patients diagnosed with heart failure, and its unique clinical and echocardiographic manifestations are described below.

\section{MATERIALS AND METHODOLOGY}

We used transthoracic echocardiography to determine the prevalence of EMF among 50 consecutive patients with heart failure, who were admitted to our hospital over a period of eight months (12.2007-08.2008).

*Address correspondence to this author at the Hospital La-Paz, Biendem, Bata, Equatorial Guinea; Tel: 972-50-2062718; Fax: 9729-742-9981; E-mail: amineuberger@yahoo.com
We compared the demographic data, clinical features and eosinophilic counts in patients with EMF and patients with other forms of heart failure, both in percentages and absolute numbers. Continuous variables were reported as means \pm standard deviation. Comparison between groups was performed by independent sample t-test. $\mathrm{p} \leq 0.05$ was considered statistically significant. All statistical analysis was done with the use of SPSS 15.0 software (SPSS Inc. Chicago, IL).

\section{RESULTS}

During the study period 50 patients were diagnosed with heart failure. Ten (20\%) had right sided EMF according to clinical and echocardiographic diagnostic criteria recently published [2]. Other causes of heart failure included pure dilated cardiomyopathy in 15 patients $(30 \%)$, dilated cardiomyopathy with partial non-compacted left ventricle in 9 $(18 \%)$, valvular pathology in $13(26 \%)$, and pulmonary hypertension with right-sided heart failure in 3 patients $(6 \%)$.

As expected, most patients with EMF were children or young adults, with an average age of $20.2 \pm 12.6$ years (range 12-51). The average age of patients with other forms of heart failure was 53.2 \pm 20.8 (range 11-78, $\mathrm{p}<0.01$ for the comparison). Six out of the ten patients with EMF were female $(60 \%)$. Among the patients with other causes of heart failure $36 \%$ were females. 
All patients presented with dyspnea, large amount of ascitis, hepatomegaly and various degrees of peripheral edema. Rapid atrial fibrillation was observed in 7 patients (70\%). Enormously dilated right atrium (RA) with average diameter of $8.8 \pm 1.2 \mathrm{~cm}$ and small obliterated RV apex with various retractions (RV apical notch - the hallmark of the disease) were observed in all patients. Ventricular valve dysfunction - free tricuspid regurgitation, resulting from the adhesion of the valvular apparatus to the ventricular wall, and paradoxical movement of interventricular septum - was also observed. In one of the cases thrombi were present in the right atrium. No cases of left sided or biventricular disease were observed.

Patients with EMF had mild anemia (average hemoglobin $12.0 \pm 1.1 \mathrm{~g} / \mathrm{dl}$ ) and eosinophilia (average absolute eosinophil count $0.51 \pm 0.29 \times 10^{9} / 1,11.9 \%$ of total leukocyte count). No statistically significant differences were found in hemoglobin and eosinophil counts between patients with EMF and patients with other forms of heart failure (data not shown). The average time from the onset of symptoms to diagnosis was $6.1 \pm 2.0$ years (range 3-9 years).

Seven patients with EMF were screened for evidence of parasitic infections. Three had evidence of parasitic infections; one with microfilaria in peripheral blood and a positive serology for schistosomiasis, and two with Trichuris trichuria in stool samples.

\section{DISCUSSION}

According to our data, right-sided EMF is widely prevalent in Equatorial Guinea and accounts for $20 \%$ of all patients with heart failure referred to our hospital. According to previously published reports, the disease affects exclusively the right ventricle in $10-28 \%$ of the patients with EMF $[2,5]$. It is thus statistically unlikely that the existence of only right-sided EMF in Equatorial Guinea is coincidental. In all probability EMF is a heterogeneous disease, or a group of closely related diseases. The exclusive involvement of the right ventricle in 10 consecutive patients hints towards a specific variant of the disease in Equatorial Guinea.

Another unique feature of EMF in our patients is the long interval between the onset of symptoms and the diagnosis, with an average of 6.1 years. The survival rate of patients with all forms of EMF after the onset of symptoms was previously reported to be around 2 years [3]. Prognosis of right-sided EMF has not been specifically studied before. It is not clear whether right-sided EMF in Equatorial Guinea represents a different disease, or if the difference in prognosis results solely from the sparing of the left ventricle.

The incidence of EMF is highest in Sub-Saharan Africa $[1,4-6]$. It is therefore tempting to assume that the etiology of EMF is related to tropical parasitic infection or a local environmental factor - although such a link has never been conclusively proved [7-11]. Three out of the ten patients with EMF in our series showed evidence of parasitic infections (one with filariasis and a positive serological test for schistosomiasis and two with Trichuris trichuria intestinal infection). Eosinophil count, though high, was not statistically different between patients with EMF and patients with other forms of heart failure. Filariasis, schistosomiasis, intestinal parasites and eosinophilia are all very common in Equatorial Guinea, and it is difficult to establish a clear cause and effect relationship between their occurrence and EMF. Other causes of right heart failure (e.g. pulmonary hypertension resulting from schistosomiasis or untreated valvular valve disease) are also common in tropical areas. Echocardiography should therefore be used to diagnose EMF as described elsewhere [2, 12-14]. We performed an echocardiographic screening program of 100 children aged 68 years, and no case of EMF was detected. It is probable that only a large prospective study of the prevalence of various parasitic infections and environmental factors in schoolchildren, and the later occurrence of EMF, will finally make it possible to reveal the cause of this common cardiomyopathy.

\section{CONCLUSIONS}

EMF is a common cause of heart failure in Equatorial Guinea. Out of fifty patients with heart failure referred to our hospital, ten had right-sided EMF. A direct link between active parasitic infections and EMF was not found. The exclusive involvement of the right ventricle in ten consecutive patients, and the relatively long survival after the onset of symptoms, hint towards a specific variant of the disease in Equatorial Guinea.

\section{REFERENCES}

[1] Bukhman G, Ziegler J, Parry E. Endomyocardial Fibrosis: Still a Mystery after 60 Years. PLoS Negl Trop Dis 2008; 2(2): e97. doi:10.1371.

[2] Mocumbi AO, Ferreira MB, Sidi D, Yacoub MH. A Population Study of Endomyocardial Fibrosis in a Rural Area of Mozambique. N Eng J Med 2008; 359: 43-9.

[3] D'Arbela PG, Mutazindwa T, Patel AK, Somers K .Survival after first presentation with endomyocardial fibrosis. Br Heart J 1972; 34: 4037.

[4] Shaper AG. The geographical distribution of endomyocardial fibrosis. Pathol Microbiol (Basel) 1970; 35: 26-35.

[5] Connor DH, Somers K, Hutt MS, Manion WC, D'Arbela PG. Endomyocardial fibrosis in Uganda (Davies' disease). An epidemiologic, clinical, and pathologic study. Am Heart J 1967; 74: 687-709.

[6] Kutty VR, Abraham S, Kartha CC. Geographical distribution of endomyocardial fibrosis in south Kerala. Int J Epidemiol 1996; 25: 1202-7.

[7] Patel AK, D'Arbela PG, Somers K. Endomyocardial fibrosis and eosinophilia. Br Heart J 1977; 39: 238-41.

[8] Falase AO. Are eosinophils the cause of endomyocardial fibrosis in the tropics? Afr J Med Med Sci 1985; 14: 1-2.

[9] Shaper AG. What's new in endomyocardial fibrosis? Lancet 1993; 342: 255-6.

[10] Andy JJ, Ogunowo PO, Akpan NA, Odigwe CO, Ekanem IA, Esin RA. Helminth associated hypereosinophilia and tropical endomyocardial fibrosis in Nigeria. Acta Trop 1998; 69: 127-40.

[11] Valiathan SM, Kartha CC. Endomyocardial fibrosis-the possible connection with myocardial levels of magnesium and cerium. Int $\mathrm{J}$ Cardiol 1990; 28: 1-5.

[12] Berensztein CS, Piñeiro D, Marcotegui M, Brunoldi R, Blanco MV, Lernan J. Usefulness of echocardiography and Doppler echocardiography in endomyocardial fibrosis. $\mathrm{J}$ Am Soc Echocardiogr 2000; 13: 385-92.

[13] Salemi VMC, Rochitte CE, Barbosa MM, Mady C. Clinical and echocardiographic dissociation in a patient with right ventricular endomyocardial fibrosis. Heart 2005; 91: 1399-99. 
[14] Hassan WM, Fawzy ME, Al Helaly S, Hegazy H, Malik S. Pitfalls in diagnosis and clinical, echocardiographic, and hemodynamic findings in endomyocardial fibrosis. Chest 2005; 128 : 3985-92.

Received: February 04, 2009

Revised: May 13, 2009

Accepted: June 01, 2009

(C) Gergana et al.; Licensee Bentham Open.

This is an open access article licensed under the terms of the Creative Commons Attribution Non-Commercial License (http://creativecommons.org/licenses/by$\mathrm{nc} / 3.0 /$ ), which permits unrestricted, non-commercial use, distribution and reproduction in any medium, provided the work is properly cited. 